\title{
Predicting the Effects of Surface Suction and Blowing on the Strouhal Frequencies in Vortex Shedding
}

\begin{abstract}
Ruben D. COHEN
Through the use of scaling arguments, a simple model describing the boundary layer behavior during vortex shedding is proposed. The main focus of the model, which is based on the assumption that the viscous diffusion length is directly proportional to the boundary layer thickness, is set towards predicting the salient features, but not the details, of the periodic flow. Promising results have been achieved upon applying the model to the classical problem of vortex shedding over a solid circular cylinder. It is noted, however, that there is a need for futher scrutiny in terms of a detailed comparison of the predicted results with existing numerical data. The model has also been applied towards investigating the behavior of the vortex shedding or Strouhal frequencies as influenced by boundary layer suction and injection (blowing) occurring normal to the stream. Among the interesting findings dealing with this part of the study, a critical Reynolds number is predicted which acts as the transition from one limiting frequency behavior to another.
\end{abstract}

Key Words : Vortex Shedding, Strouhal Number, Suction, Injection

\section{Introduction and Overview}

The behavior of unsteady flow over solid bodies is a subject of active research emphasizing on fluidstructure interactions in aero-and hydrodynamics. The existing literature related to this topic is quite extensive, and therefore presenting an in-depth description here is found to be unnecessary. For convenience, however, a brief overview of some of the important and well known characteristics associated with vortex shedding over bluff bodies is included in the following paragraphs obtained from Rothberg ${ }^{(1)}$.

For an isolated and stationary solid circular cylinder being subjected to low Reynolds number flow (based on the cylinder diameter), a symmetrical streamline pattern about the body with no flow separation is established. Onset of separation begins at a Reynolds number of 5 to 6 after which instabilities caused by the amplification of Tollmien-Schlichting waves $^{(2)}$ start to develop at a Reynolds number of

* Received 9th July, 1990

** Dept. of Mechanical Engineering \& Materials Science, Rice University, Houston, Texas 77251 about 40 . These, ultimately, cause vortices to shed in a periodic manner over the surface of the body. An interesting observation made by Gerrard ${ }^{(3)}$ is that when the shear layers are brought closer together (i. e. thinner boundary layers), as would occur with the motion of the separation points towards each other, the frequency of vortex shedding is increased.

The flow about the cylinder and throughout the wake remains laminar up to a Reynolds number of approximately 150 , after which a transition to turbulence in the wake begins to develop. As the Reynolds number increases beyond this value, the transition to turbulence in the wake continues to grow and moves further upstream toward the cylinder boundary layer. The flow regime ranging from the onset of wake turbulence to the transition to turbulence in the cylinder boundary layer is known as the subcritical range. Critical flow occurs at approximately $R e_{d}=3 \times 10^{5}$. This is accompanied by a large reduction in the drag coefficient as the separation points move rearward on the cylinder. Flows between the critical value and a Reynolds number of approximately $3.5 \times 10^{6}$ show no organized pattern in the wake, and vortex shedding is not present. Depending on the reference, flows at $R e_{d}$ 
greater than $3 \times 10^{5}$ are called either supercritical or transcritical. $R e^{-e s t a b l i s h m e n t ~ o f ~ v o r t e x ~ s h e d d i n g ~ i s ~}$ observed at Reynolds numbers exceeding $3.5 \times 10^{6}$. Fig. $1^{(4)}$ summarizes the results discussed above. Here the Strouhal number, $S t$, which is defined by

$$
S t=f d / U_{\infty}
$$

where $f$ is the frequency of shedding, $d$ being the cylinder diameter, and $U_{\infty}$ representing the free stream velocity, is plotted against Reynolds number, $R e_{d}$, where

$$
R e_{d}=U_{\infty} d / \nu
$$

( $\nu$ is the kinematic viscosity of the fluid). A more detailed analysis of the characteristic features observed here are discussed in a later section of this paper.

It is now important to focus briefly on the analytical investigations of these problems that began in the form of stability analysis of vortex streets ${ }^{(5)}$, and have, over a period of many years, been extended to detailed numerical simulations of the unsteady flow fields over cylindrical bodies ( 1 and 6 among a great many others). In reference to the many numerical investigations conducted throughout the past few years, it is notable that rather superb agreements with the experimental observations discussed above have been achieved.

In many instances, especially where practical engineering designs are involved, it is somewhat desirable to bypass the use of extensive computations and instead rely on scaling parameters for characterizing the prominent features often associated with the specific problem of interest. With this in mind, a simple model with the sole purpose of establishing a means for quick predictions of certain aspects of vortex shedding over bluff bodies is proposed here. Being principally based on scaling arguments and on a relatively simple assumption relating the viscous diffusion length and the boundary layer thickness, the model aims at providing a straightforward technique for seeking the important dimensionless parameters

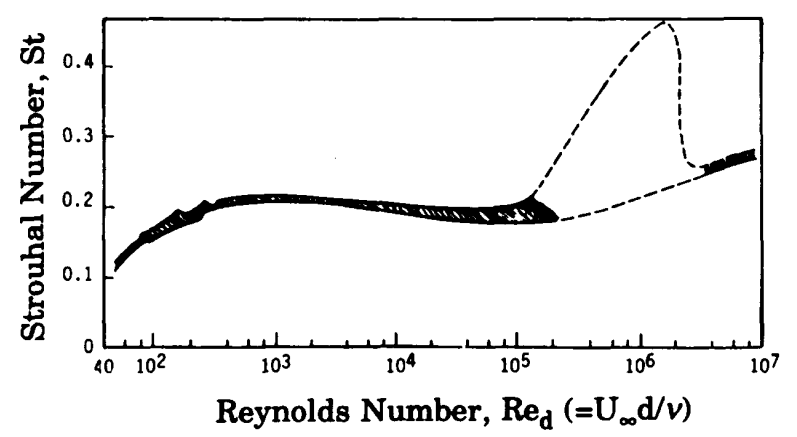

Fig. 1 Characteristic features of the Strouhal number versus Reynolds number for flow over a circular cylinder (from Ref. (4), with permission). that may prove valuable for modeling and design purposes.

\section{Analysis of the $S t-R e_{d}$ Relationship}

Referring back to Fig. 1 and to the discussions preceding it, one may subdivide the results into the following 4 catagories :

I $40<R e_{d}<150$

laminar boundary layer \& wake

II $150<R e_{d}<3 \times 10^{5}$

laminar boundary layer, turbulent wake $(2 \cdot b)$

III $3 \times 10^{5}<R e_{d}<3.5 \times 10^{6}$

no observed vortex shedding

IV $R e_{d}>3.5 \times 10^{6}$

vortex shedding, turbulent wake $\&$ boundary layer

Furthermore, we choose to characterize the $S t-R e_{d}$ plot by the following power law relation:

$$
S t \sim \operatorname{Re}_{d}^{\beta}
$$

where the value of the power coefficient $\beta$ varies with the different regimes of $R e_{d}$ defined in Eqs. (2). Using the results of Fig. 1 and from other more detailed graphical data (i. e. Fig. 2.9 in Ref. (7)), it can be shown that

$\begin{array}{ll}\beta \approx 0.2 & \text { Region I } \\ \beta \approx 0 & \text { Region II } \\ \beta \approx 0.1 & \text { Region IV }\end{array}$

which interestingly suggest that the Strouhal number is overall very weakly dependent on the Reynolds number within the ranges investigated. Although not generally presented in the form given by Eqs. ( 3 ) and (4), this conclusion is very much recognized and well accepted among the fluid dynamics community.

With this rather peculiar result in mind, we shall now proceed to develop a simple model which incorpo-

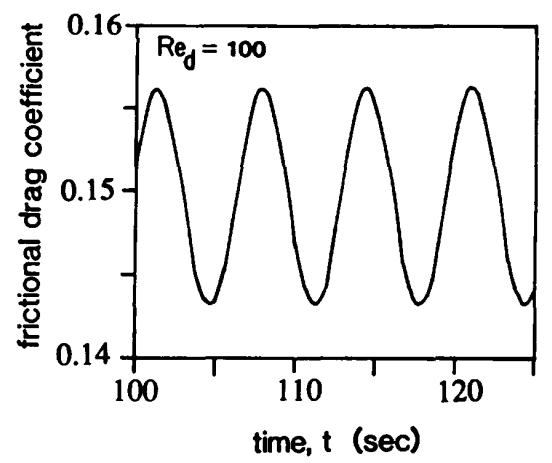

Fig. 2 Time dependent behavior of the frictional drag coefficient for flow at $R e_{d}=100$ over a circular cylinder (from Ref. (6), with permission). Here, frictional drag is calculated by integrating the numerically obtained wall shear stress from the forward stagnation $(\theta=\pi)$ to the point of separation along the upper boundary layer. 
rates the conservation principles of mass and momentum to describe the boundary layer behavior during vortex shedding. The ultimate goal for the model is, of course, to predict this quasi-independent characteristic of the Strouhal number. Once this is achieved, then applications to a wider range of problems can be made. These would include, for example, predictions of the behavior of vortex shedding frequencies over porous bluff bodies with suction and injection occurring on the surface, normal to the flow.

It is now important to stress the fact that the phenomenon of vortex shedding, particularly in the presence of boundary layer suction and blowing, is an extremely complex one whose details can be investigated only by rigorous numerical or experimental techniques. The very simple model which follows in the next section should by no means be viewed as an attempt to establish the exact behaviors. Rather, it should just be considered as an order of magnitude approach set towards determining only the characteristic behaviors of the Strouhal frequencies in the certain asymptotic limits to be discussed shortly.

\section{Model Development}

This work is divided into two parts. Part [1] deals with vortex shedding over impermeable cylinders, and part [2] accounts for the influence of boundary layer suction and blowing on the Strouhal frequencies.

[1] Vortex shedding over an impermeablecylinder: Although derivation of the model for the impermeable cylinder is readily availabe in existing litera ture ${ }^{(8)}$, a brief review is included here for convenience to the reader.

It is known that thin boundary layers over solid bodies are generally characteristic of high Reynolds number flows. Additionally, in the presence of an adverse pressure gradient, flow separation and vortex formation inevitably occurs. With the free stream usually being irrotational and the wake, rotational, by Helmholtz's theorem we can conclude that the vorticity contained in the wake must originate from within the boundary layer.

We now propose that in the presence of vortex shedding, the primary mechanism of vorticity transport from boundary layer to the wake is due to vortex shedding. Hence, upon detachment and shedding, the vortex, having been formed in the separated region of the flow in the boundary layer, shall carry with it part of the boundary layer, and consequently its characteristics, and move downstream at a speed approximately equal to the free stream yelocity, $U_{\infty}$, to eventually form the wake. This mechanism will therefore lead to a partial removal of the boundary layer, hence causing its periodic collapse and regrowth. Evidence of this is presented by the periodic oscillation of the frictional drag along the surface of the cylinder, as illustrated in Fig. 2 for $R e_{d}=100$. Here, drag is calculated by integrating the numerically obtained wall shear stress from the forward stagnation $(\theta=\pi)$ to the point of separation along the upper boundary layer ${ }^{(6)}$. Further proof is provided after observing that the natural shedding of vortices also induces small increases of the heat transfer rate in the attached flow region ${ }^{(9)}$.

The approach towards developing the model mainly involves the assumption that the viscous diffusion length is directly proportional to the boundary layer thickness. We shall now investigate the implications of this hypothesis by applying scaling arguments to analyze the incompressible NavierStokes Equation. In reference to Fig. 3, we refer to the $\theta$ or azimuthal component of the polar Navier-Stokes Equation given below :

$$
\begin{aligned}
\frac{\partial u}{\partial t} & +\frac{u}{r} \frac{\partial u}{\partial \theta}+v \frac{\partial u}{\partial r}+v \frac{u}{r}=-\frac{1}{\rho r} \frac{\partial p}{\partial \theta} \\
& +\nu\left\{\frac{\partial^{2} u}{\partial r^{2}}+\frac{1}{r} \frac{\partial u}{\partial r}-\frac{u}{r^{2}}+\frac{1}{r^{2}} \frac{\partial^{2} u}{\partial \theta^{2}}+\frac{2}{r^{2}} \frac{\partial v}{\partial \theta}\right\}
\end{aligned}
$$

where $u$ and $v$, respectively, denote the tangential and the normal velocity components, and $p$ is the local pressure. Considering very thin boundary layers, i. e. $\delta / R \ll 1$ with $\delta$ being the characteristic boundary layer thickness over the body, Eq. ( 5 ) simplifies to the well known unsteady boundary layer equation given by:

$$
\frac{\partial u}{\partial t}+\frac{u}{r} \frac{\partial u}{\partial \theta}+v \frac{\partial u}{\partial r}=\frac{U(\theta)}{r} \frac{\partial U(\theta)}{\partial \theta}+\nu \frac{\partial^{2} u}{\partial r^{2}}
$$

where $U(\theta)$ is the free stream velocity at the edge of

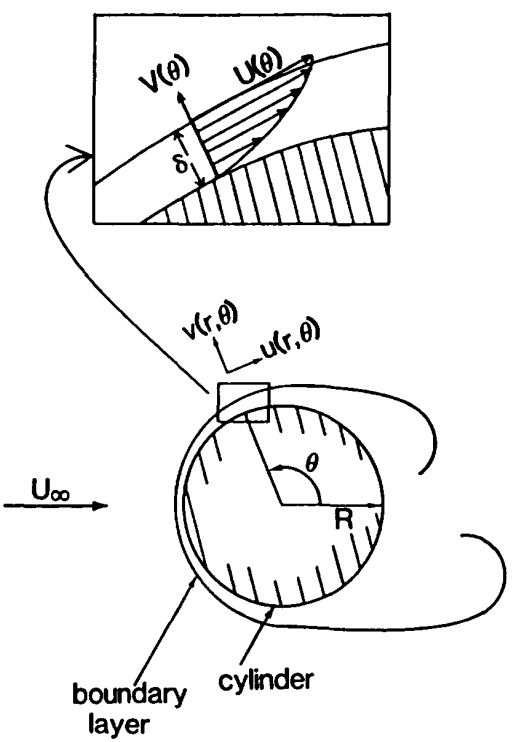

Fig. 3 Schematic of flow and vortex shedding over a circular cylinder (from Ref. (8), with permission). 
the boundary layer. Eq. (6) results after realizing that for boundary layers

$$
\frac{\partial \theta}{\partial_{r}} \approx 0
$$

so that

$$
\frac{1}{\rho r} \frac{\partial p}{\partial \theta}=-\frac{U(\theta)}{r} \frac{\partial U(\theta)}{\partial \theta}
$$

Eq. $(7 \cdot \mathrm{b})$ is simply the $\theta$-direction pressure gradient obtainable from the inviscid flow relationship, depending on the shape of the solid body. Order of magnitude scaling of Eq. ( 6 ) gives

$$
\frac{\partial u}{\partial t}+K \frac{U^{2}}{R} \sim \frac{U^{2}}{R}+\nu \frac{U}{\delta^{2}}
$$

where $R$ is the characteristic size of the body (i. e. $R$ $=d / 2$ for a circular cylinder of diameter $d$ ), and $K$ is a dimensionless constant of order unity implemented to distinguish between the convective acceleration and the pressure terms appearing, respectively, on the left hand side and right hand side of Eq. (6).

At this point we shall investigate the consequence of our assumption that the governing mechanism of vortex shedding is the periodic formation, detachment, and removal of part of the boundary layer, along with the vortex. This, therefore, implies that the boundary layer thickness, $\delta$, is periodically dependent on time, i. e.

$$
\delta \sim \delta(t)
$$

where $0 \leq t<\tau, \tau \leq t<2 \tau$, etc. with $\tau$ being the period of shedding.

As a result of the somewhat sudden removal and the subsequent partial collapse of the viscous boundary layer at the time of shedding, $\delta$ becomes small enough for the viscous term in Eq. ( 8 ) to dominate over the convective term, $U^{2} / R$. Hence

$$
\nu \frac{U}{\delta^{2}} \gg \frac{u^{2}}{R}
$$

which implies that corresponding to time $t / \tau \ll 1$, the following will govern :

$$
\frac{\partial u}{\partial t} \approx \nu \frac{\partial^{2} u}{\partial r^{2}}
$$

In other words, immediately after detachment of the vortex and the subsequent collapse of the boundary layer, its growth begins by the process of viscous diffusion characterized by Eq. (11). This diffusive growth continues until, more or less, a time of the order of magnitude given by the period of shedding, $\tau$. At this time when the vortex has almost grown to its maximum and steady size and ready to detach, convective acceleration will begin to prevail over the unsteady term. This suggests that

$$
\frac{\partial u}{\partial t} \ll \frac{U^{2}}{R}
$$

at $t / \tau \sim 1$. As a result of this, Eq. ( 6 ) reduces to

$$
\frac{u}{r} \frac{\partial u}{\partial \theta}+v \frac{\partial u}{\partial r} \approx \frac{U(\theta)}{r} \frac{\partial U(\theta)}{\partial \theta}+\nu \frac{\partial^{2} u}{\partial r^{2}}
$$

which is the steady state boundary layer equationassumed to be reached at time $t \sim \tau$.

Based on classical scaling arguments, it follows from Eqs. (11) and (13), respectively, that

$$
\delta(t) \sim \sqrt{\nu t} \quad \text { for } \quad 0 \leq t<\tau
$$

and

$$
\delta(t) \sim \sqrt{\frac{\nu R}{U}} \quad \text { when } \quad t \sim \tau
$$

It should be mentioned that all numerical constants have been excluded up to this point so that we can focus primarily on providing the important characteristic or asymptotic behaviors and the scaling parameters involved. Finally, we expect Eqs. (14) and (15) to approach one another in their orders of magnitude at time $t \approx \tau, i$. $e$.

$$
\sqrt{\nu \tau} \sim \sqrt{\frac{\nu R}{U}}
$$

Thus, by virtue of this argument, we get

$$
\tau \sim \frac{R}{U}
$$

after solving for the shedding period, $\tau$. But within a constant, Eq. (17) can be re-written in terms of the cylinder diameter, $d$, free stream velocity, $U_{\infty}$, and the shedding frequency, $f$ (where $f \equiv 1 / \tau$ ), i. e.

$$
\frac{1}{f} \sim \frac{d}{U_{\infty}}
$$

Now, upon defining the dimensionless Strouhal number, $S t$, by

$$
S t \equiv \frac{f d}{U_{\infty}}
$$

we find that our proposed model leads to

$$
\text { St } \sim \text { constant }
$$

by means of Eq. (18). This result simply states that the Strouhal number is a constant, independent of the Reynolds number, which, surprisingly, is quite in satisfactory agreement with experimentally obtained $S t$ $R e_{d}$ relationships for various body shapes ; an example of one being represented in Fig. 4 obtained from Ref. ( 7 ). We find it important to point out that since the model is based on the application of NavierStokes Equation to the boundary layer only, it is expected to be valid so long as the boundary layer, but not necessarily the wake, remains laminar. Also notable is that, in reality, slight dependencies, such as those given by Eqs. ( 3 ) and (4) for the case of a circular cylinder of the Strouhal on the Reynolds number, do tend to exist. With relation to our model, however, this discrepancy appears to be reasonably small, especially after considering our extremely simplistic approach to the problem. Altogether, we conclude that the model does provide us with a physical view that is seemingly consistent with exgerimental data. 


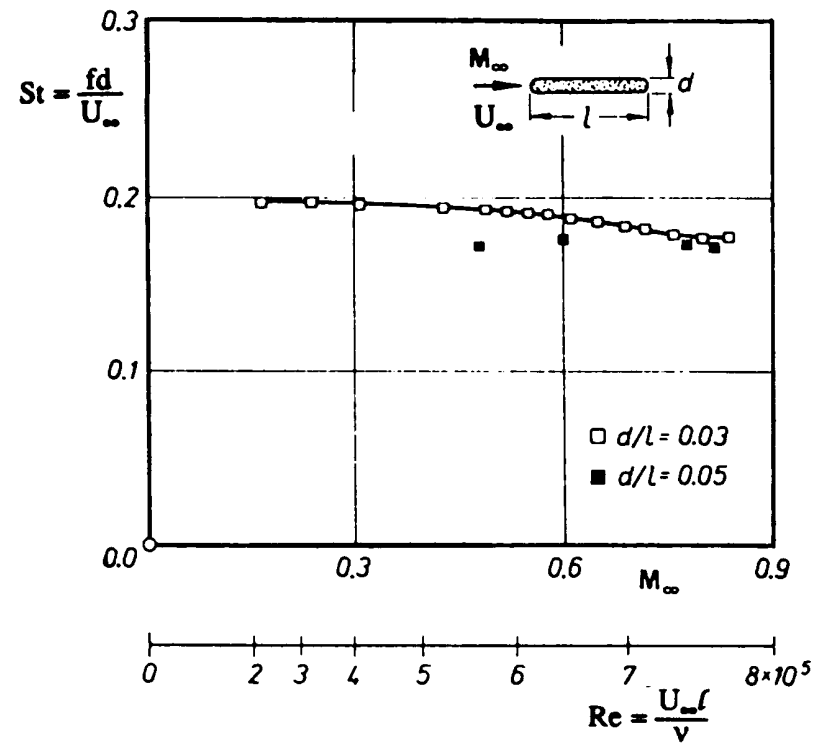

Fig. 4 Strouhal voersus Reynolds number for the vortex street behind a flat plate at zero incidence (from Ref. ( 7), with permission).

[2] Effect of Normal Surface Suction \& Injection on the $S t-R e_{d}$ Relationship

In the sections that follow, we use the proposed physical model in an attempt to predict the characteristic behavior of the $S t-R e_{d}$ relationship during vortex sheddng. Fig. 5 shows a typical body, in this case being a cylinder of diameter $d$, being subjected to flow approaching with free stream velocity $\dot{U}_{\infty}$, and having fluid properties $\rho$ and $\mu$. Here, the cylinder is assumed to be porous, with fluid being sucked in or injected out with uniform velocity (suction or injection flow rate per unit surface area), $v_{0}$, normal to the surface.

The character of the steady state boundary layer, $\delta$, as affected by suction and injection, is schematically illustrated for the flat plate configuration in Fig. 6. For simplicity, we use the flat plate here with the realization that the asymptotic, but not exact, behaviors can be extended to other geometries, such as circular cylinders. Using the simple Von Karman momentum integral approach, we can demonstrate that near the leading edge both curves (suction and injection) asymptotically approach the Blasius behavior characterizd by $\delta \sim \sqrt{\nu L / U_{\infty}}$, with $L$ being the characteristic plate length. Further downstream, however, the curves approach their specific characteriscs of $\delta \sim v_{0} L / U_{\infty}$ and $\delta \sim \nu / v_{0}$, for normal injection and suction, respectively. For other porous surfaces where streamwise pressure gradients are not zero, these behaviors can still be shown to be directly analogous to the above from an order of magnitude analysis of the boundary layer equation given by Eq. ( 6 ). In this

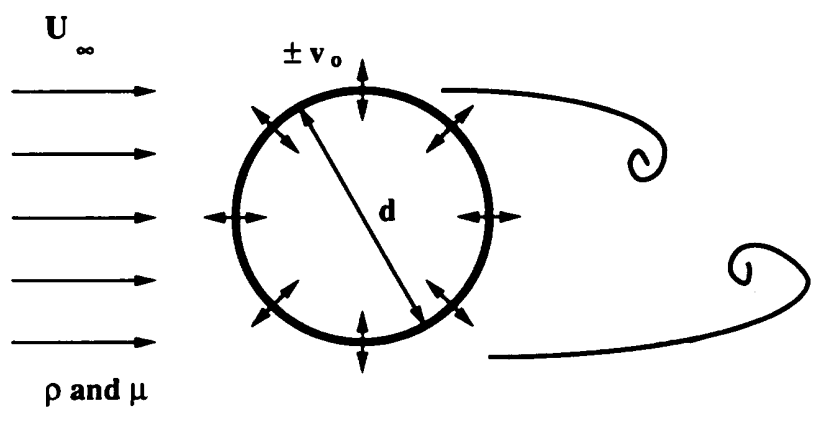

Fig. 5 Schematic of flow and vortex shedding over a circular cylinder with surface suction $\left(-v_{0}\right)$ or surface injection $\left(+v_{0}\right)$.

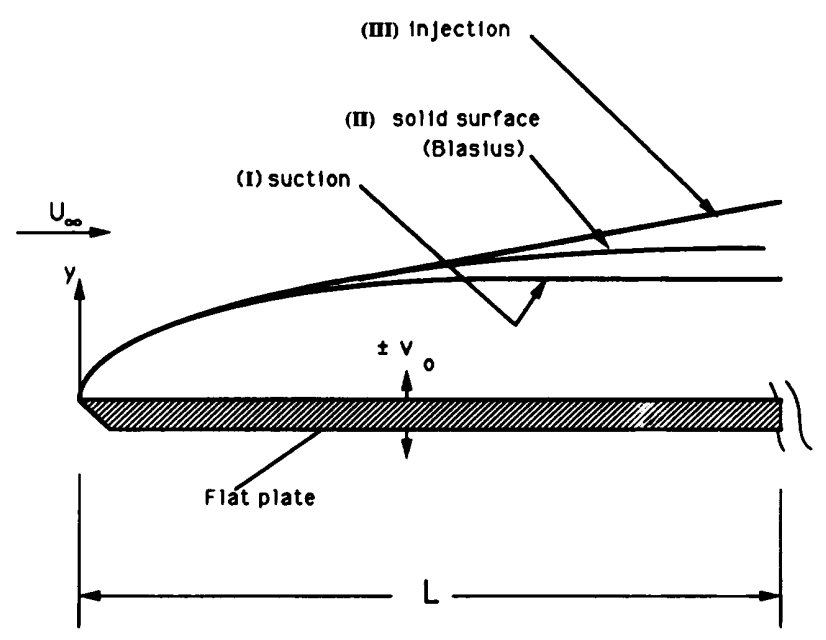

Fig. 6 Characteristic behaviors of the boundary layers formed in the presence of surface suction (curve I), surface injection (curve III), and impermeable surface (curve II).

situation, we must take the velocity boundary condition on the surface as

$$
[\vec{V}]_{\text {surface }}= \pm \dot{i}_{r} v_{0}
$$

(where + and - , respectively, denote injection and suction) instead of the zero used for the impermeable circular cylinder problem. As a result, applying scaling arguments to Eq. ( 6 ) yields

$$
\frac{\partial u}{\partial t}+K \frac{U_{\infty}^{2}}{d}+v_{0} \frac{U_{\infty}}{\delta} \sim \frac{U_{\infty}^{2}}{d}+\nu \frac{U_{\infty}}{\delta^{2}}
$$

The difference between Eq. ( 8 ) (for an impermeable surface) and Eq. (22) is that the latter accounts for the effect of normal suction and injection.

In reference to the above equation, we shall now derive the characteristics of the boundary layer thickness at steady state, i. e. $\partial u / \partial t=0$. This result is necessary for later utilization in model development.

(i) Suction-Considering suction normal to the surface, we realize that there basically exist ( 2 ) distinctive boundary layer structures, as shown in Fig. 6 . The 
initial portion is the "Blasius" type (i. e. "Blasius" type boundary layer is used in reference to steady boundary layers over impermeable objects) which occurs near the leading edge where the effect of suction is not yet felt by the flow. The influence of suction, however, begins to show further downstream where, as will be shown later, the boundary layer thickness approaches a constant value over the remainder of the plate.

Returning to near the leading edge where the Blasius structure dominates, this essentially occurs when

$$
v_{0} \frac{U_{\infty}}{\delta} \ll \frac{U_{\infty}^{2}}{d}
$$

or simply

$$
\frac{v_{0} d}{\delta U_{\infty}} \ll 1
$$

corresponding to when the effect of suction is negligible. Hence, it obviously follows that under the condition dictated by Eq. (24), we get the "Blasius" type given by

$$
\frac{\delta}{d} \sim \sqrt{\frac{\nu}{U_{\infty} d}}
$$

just as in Eq. (15).

In the other extreme of Eq. (24) we have the boundary layer structure as affected by normal suction (constant $\delta$ )

$$
\frac{v_{0} d}{\delta U_{\infty}} \gg 1
$$

which, from Eq. (22) at steady state, leads to

$$
\frac{v_{0} U_{\infty}}{\delta} \sim \frac{\nu U_{\infty}}{\delta^{2}}
$$

or simply

$$
\delta \sim \frac{v}{v_{0}}
$$

thereby proving that the characteristic thickness of the boundary layer, in the presence of strong surface suction, approaches a constant value along the down. stream section of the body. Substitution of the above in Eq. (26), therefore, yields the condition for which Eq. (28) maintains its validity. Hence,

$$
\delta \sim \frac{\nu}{v_{0}} \quad \text { when } \frac{v_{0}^{2} d}{\nu U_{\infty}} \gg 1
$$

Upon defining $R e^{*}$ as

$$
R e^{*} \equiv v_{0} d / \nu
$$

we re-write Eq. (29) as

$$
\frac{\delta}{d} \sim \frac{1}{R e^{*}} \quad \text { when } \quad \frac{R e^{* 2}}{R e_{d}} \gg 1
$$

Now, in accordance with our shedding model, we follow the assumptions presented in Eq. (14) to write

$$
\frac{\delta}{d} \sim \frac{\sqrt{\nu \tau}}{d}
$$

where once again $\tau$ denotes the shedding period, which is just the time scale needed for the boundary layer to achieve its steady state value (Eq. (29) for the case of suction). Equating Eqs. (31) and (32) and solving for $\tau$ yields

$$
\tau \sim \frac{d^{2}}{\nu} \frac{1}{R e^{* 2}}
$$

In terms of the more useful Strouhal number, the above reduces to

$$
[S t]_{\text {suction }} \equiv \frac{f d}{U_{\infty}} \sim \frac{R e^{* 2}}{R e_{d}} \quad \text { when } \quad \frac{R e^{* 2}}{R e_{d}} \gg 1
$$

where $f$ is just $\tau^{-1}$. The above implies that $S t$ increases with increasing rate of suction.

(ii) Blowing-We refer again to Eq. (22) at steady state for analyzing the boundary layer character as affected by blowing normal to the surface. Once more, the boundary layer possesses 2 distinctive behaviors. One being the Blasius type manifested in Eq. (25), which results near the leading edge where the effects of blowing are insignificant, i. e. when Eq. (24) is satisfied. Far from the leading edge, however, structural transition occurs owing to the fact that in this region, due to continuous surface blowing with arbitrary rate of $v_{0}$, the boundary layer thickness growns linearly and eventually becomes large enough to render

$$
v_{0} \frac{U_{\infty}}{\delta} \gg \frac{\nu U_{\infty}}{\delta^{2}}
$$

or

$$
\frac{\delta}{d} \gg \frac{1}{R e^{*}}
$$

simply because the RHS of Eq. (35) decays with $\delta^{-2}$ while the LHS is proportional to $\delta^{-1}$. Thus for normal injection, we obtain the steady state behavior of $\delta$ through the relationship

$$
\frac{U_{\infty}^{2}}{d} \sim \frac{v_{0} U_{\infty}}{\delta}
$$

obtainable from Eq. (22) for $\partial u / \partial t=0$ and the condition of Eq. (35). The above implies that the inertial terms have the same orders of magnitude. Eq. (37) is simplified to yield

$$
\frac{\delta}{d} \sim \frac{v_{0}}{U_{\infty}}
$$

which is the steady state characteristic of the boundary layer thickness as affected by injection. Thestructure of Eq. (38) can be easily verified by applying the von Karman momentum integral on a boundary layer with injection, after satifying the criterion given by Eq. (26), i. e. far from the leading edge where Blasius is no longer valid.

With $\delta / d$ available from the above, the condition of Eq. (36) becomes

$$
\frac{R e^{* 2}}{R e_{d}} \gg 1
$$

so that for normal surface injection

$$
\frac{\delta}{d} \sim \frac{v_{0}}{U_{\infty}} \quad \text { when } \quad \frac{R e^{* 2}}{R e_{d}} \gg 1
$$

Again, according to a previous argument regarding 
the transient behavior of $\delta$ (i. e. $\delta(\tau) \sim \sqrt{\nu \tau}$ ), we get for injection

$$
\begin{aligned}
& \frac{\sqrt{\nu \tau}}{d} \sim \frac{v_{0}}{U_{\infty}} \quad \text { when } \quad \frac{R e^{* 2}}{R e_{d}} \gg 1 \\
& \tau \sim \frac{d^{2} v_{0}^{2}}{\nu U_{\infty}^{2}}
\end{aligned}
$$

after solving for $\tau$. Finally, in terms of the Strouhal frequency, we obtain

$$
[S t]_{\text {Injection }} \equiv \frac{f d}{U_{\infty}} \sim \frac{R e_{d}}{R e^{* 2}} \quad \text { when } \quad \frac{R e^{* 2}}{R e_{d}} \gg 1
$$

to represent our predicted asymptotic $S t-R e_{d}$ relationship in the presence of injection. Eq. (42) suggests that normal injection decreases the Strouhal frequency.

\section{Analysis of Results}

In the previous sections, we investigated the influence of surface suction and injection on the Strouhal frequency. For convenience, these results have been put together below :

$$
\begin{aligned}
& {[S t]_{\mathrm{szcction}} \sim \frac{R e^{* 2}}{R e_{d}} \quad \text { when } \frac{R e^{* 2}}{R e_{d}} \gg 1} \\
& {[S t]_{\mathrm{Injection}} \sim \frac{R e_{d}}{R e^{* 2}} \quad \text { when } \frac{R e^{* 2}}{R e_{d}} \gg 1} \\
& {[S t]_{\text {suction\&injection }} \sim \text { constant } \quad \text { when } \frac{R e^{* 2}}{R e_{d}} \ll 1}
\end{aligned}
$$

The behaviors of the equations provided above can be best visualized through the $\log -\log$ plot of $S t$ versus $R e_{d}$ portrayed in Fig. 7.

An interesting observation readily deducted from this plot is that the Strouhal numbers corresponding to normal surface suction and injection are, respectively, greater and smaller than those associated with the impermeable cylinder (i. e. $R e^{*}=0$ ). In other words, suction which acts to reduce the overall thickness of the boundary layer, tends to increase the

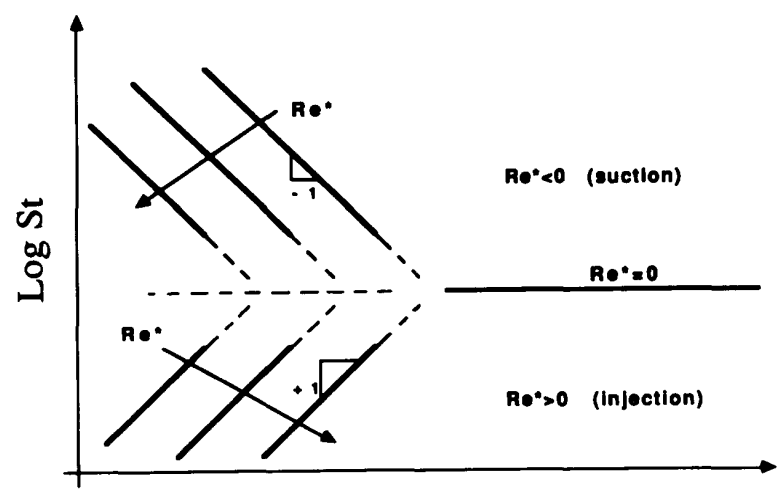

$$
\log R \mathbf{e}_{\mathrm{d}}
$$

Fig. 7 Prediction of $S t$ versus $R e_{d}$ as affected by normal suction and injection at different values of $R e^{*}$. shedding frequency above that of flow over the impermeable body, and vice versa. This finding, at least for the time being, seems to be consistent with Gerrard ${ }^{(3)}$ who observed that thinner shear (boundary) layers lead to higher shedding fsequencies. We also note from Fig. 7 that the slopes obtained from the log $(S t)$ versus $\log \left(R e_{d}\right)$ curves acquire values of +1 for injection and -1 for suction.

It is now worth commenting about the influence that suction may have on delaying, and even suppressing, the onset of flow separation in the boundary layer. This well known effect would, therefore, render our predicted asymptotic limit (slope of -1 in the log $(S t)$ versus $\log \left(R e_{d}\right)$ plot) in vortex shedding with normal suction very difficult, if not impossible, to achieve. The question of whether or not this can be reached is, at this point, left to be answered by thorough analytical and/or experimental investigations. Nevertheless, we do note that, at least, this predicted asymptote for suction dominated flows provides an upper bounds that may prove useful in practical design applications.

Another characteristic feature worth noting is that in the limit $R e^{* 2} / R e_{d} \gg 1$, the impacts of suction and injection appear to be most visible, while as $R e^{* 2} / R e_{d}$ becomes small, they lose all their effects, and the $S t-R_{d}$ curve approaches that of shedding over a solid impermeable body. This basically sug. gests that in the vicinity of

$$
\frac{R e^{* 2}}{R e_{d}} \sim 1
$$

there is a transition from one asymtpotic behavior (shedding in the presence of suction or blowing) to the other (shedding over an impermeable surface). This is exemplified in Fig. 8 for suction at a specific value of $R e^{*}$. It therefore follows that we can define a critical Reynolds number, $\left[R e_{d}\right]_{\text {critucal }}$, by letting

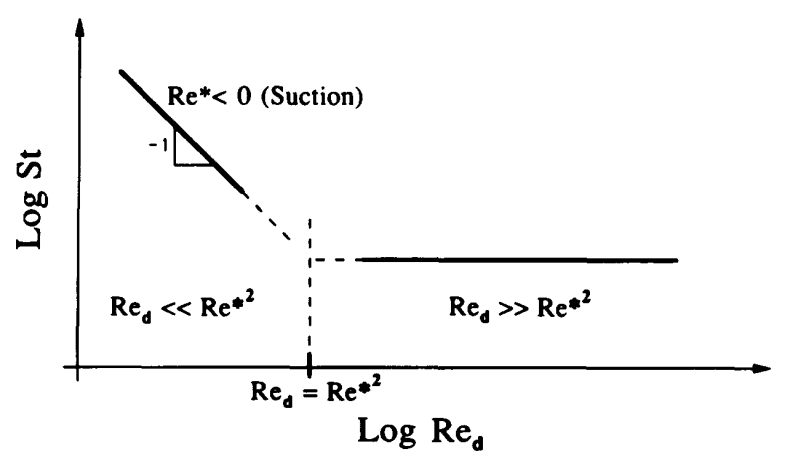

Fig. $8 S t$ versus $R e_{d}$ for normal suction at a specific value of $R e^{*}$. Important features are the asymptotic behaviors at $R e_{d} \ll R e^{* 2}$ and $R e_{d} \gg$ $R e^{* 2}$ (heavy solid lines), and the transitional region at $R e_{d}=R e^{* 2}$. 


$$
\left[R e_{d}\right]_{\text {critcal }}=R e^{* 2}
$$

which suggests that if we operate at

$$
R e_{d}<\left[R e_{d}\right]_{\text {critucal }}
$$

then the effects of suction and injection, conveniently characterized by $R e^{*}$, gain importance and, therefore, must be accounted for. Otherwise, when operating at

$$
R e_{d}>\left[R e_{d}\right]_{\text {critical }}
$$

then these effects can be neglected, and the body considered to be impermeable.

\section{Conclusions}

A simple model based on a hypothesis concerning the behavior of the boundary layer during vortex shedding is presented. Upon applying the model, which is derived using scaling arguments, to an impermeable stationary body such as the classical case of the circular cylinder, we satisfactorily predict the quasiconstant characteristics of the Strouhal frequency. Extending the model to account for the possibility of vortex shedding in the presence of surface suction and injection occurring normal to the surface leads to the results outlined below :

(i) Prediction of a critical Reynolds number, denoted here by $\left[R e_{d}\right]_{\text {critccal }}$, which is found to be equal to $\operatorname{Re}^{* 2}$ (Figs.7 and 8). This appears to serve as the transition from one characteristic asymptotic behavior to another. In other words, the effects of suction and injection are most visible when operating the system below this critical Reynolds number, whereas, they are lost when operating at Reynolds numbers higher than $\left[R e_{d}\right]_{\text {crutcal. }}$

(ii) When operating below $\left[R e_{d}\right]_{\text {crtical }}$, suction tends to increase the Strouhal frequency while injection decreases it.

(iii) On a log-log plot of $S t$ versus $R e_{d}$, at $R e_{d}$ 's below the critical value, all curves have slopes that asymptotically approach \pm 1 , where $(+)$ and $(-)$, respectively, correspond to injection and suction. Moreover, these asymptotic slopes appear to be independent of $R e^{*}$.

(iv) Finally, in reference to Eqs. (34), (42), and (43), and also from Figs. 7 and 8, we conclude that it may be more appropriate to graphically portray the effects of Reynolds number on the Strouhal number as $S t$ versus $R e^{* 2} / R e_{d}$, rather than $S t$ versus $R e_{d}$. This is more attractive because if we choose the independent parameter to be $R e^{* 2} / R e_{d}$ instead of $R e_{d}$, we can unify all the curves of Fig. 7, corresponding to different values of $R e^{*}$ (including the impermeable case of $R e^{*}$ $=0$ ), at least in the asymptotic limits of $R e^{* 2} / R e_{d} \ll 1$ and $\gg 1$ as illustrated in Fig. 9. By doing so, however, we lose all the intricate details that are embedded in the $S t-R e_{d}$ curve (i. e. Figs. 1 and 4) since only the (almost) constant or overall average value of the
Strouhal number is incorporated to represent the impermeable case given by $R e^{* 2} / R e_{d}=0$ (e.g. St $\approx 0.21$ and 0.19 , respectively, for the impermeable circular cylinder and the finite flat-shaped body shown in Fig. 4). It is also worth noting that these "average" Strouhal numbers are not significantly different for a wide variety of body shapes ${ }^{(10)}$.

Since at this time we are not aware of any experimental or numerical works dealing directly with the indicated problem, the validity of the results of Fig. 9 remains to be tested by further investigations. In connection with this, however, reference is made to Bearman's experiments ${ }^{(11)}$ on the effects of base bleed on vortex shedding frequencies. A conclusion of that work is that the Strouhal frequency first rises and then falls with increasing injection rates at the base. Altogether, the effects of base bleed on the Strouhal number seem to be insignificant, as concluded also by Wood ${ }^{(12)}$. From the configuration of the body involved in the abovementioned studies, however, we note that the boundary layer region is not subjected to any normal blowing. This, therfore, indicates that the nature of the problem considered in Refs. (11) and (12) is quite different from the one examined here, and thus the results obtained there could not be compared with those predicted here.

In addition, we note that a number of investigations concerning high Reynolds number flow on porous plates, as illustrated schematically in Fig. 10, have been conducted ${ }^{(13)(14)}$. In reference to these works which were carried out on plates of varying porosities, we note that they are somewhat related to the case of boundary layer suction since the leakage through the porous plate could very well represent an effective rate of suction. Here, it is interesting to observe that increasing the plate porosity, and thereby the suction

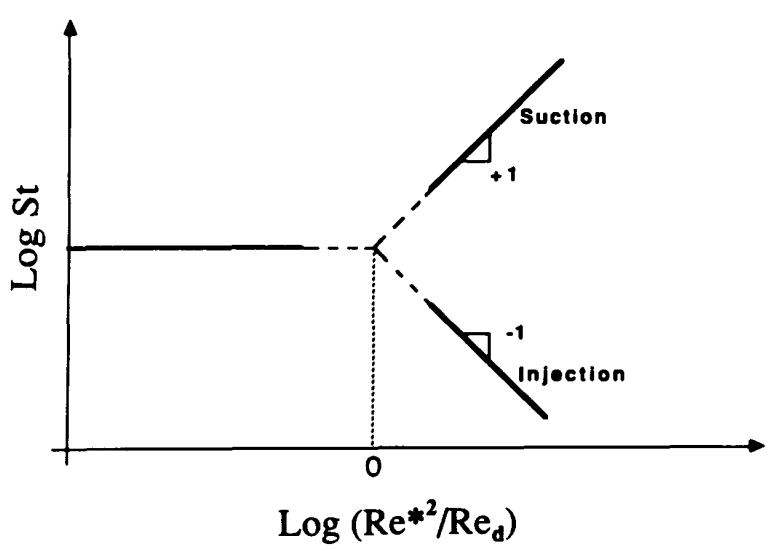

Fig. $9 S t$ versus $R e^{* 2} / R e_{d}$ emphasizing the unification of all curves obtained at different rates of normal suction and injection. 


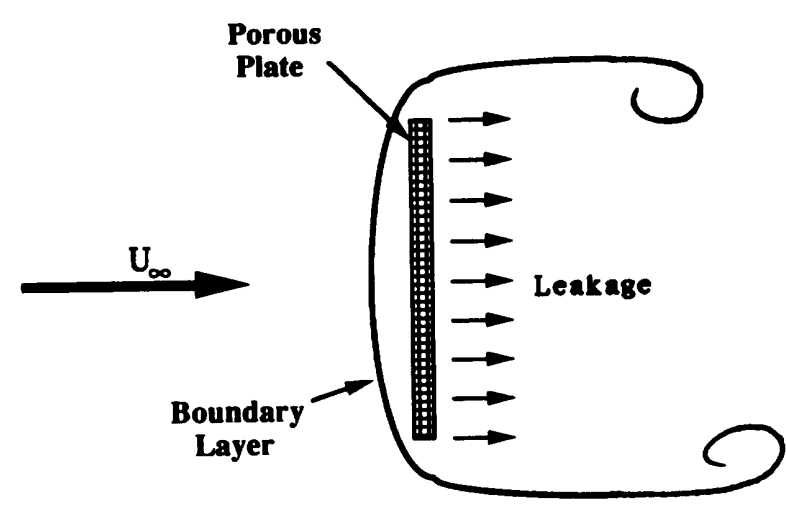

Fig. 10 Shematic diagram of high Reynolds number flow incident on a porous plate.

rate, leads to an increase in the Strouhal frequency ; a result which, qualitatively, is in good agreement with the predictions of the proposed model.

Finally, as mentioned earlier, we find it important to emphasize strongly that the model is obviously not an exact analysis, but is only proposed to provide a rather simplistic approach to predicting the effects of normal surface suction and blowing on the asymptotic limits of vortex shedding frequencies.

\section{References}

(1) Rothberg, R., "A Numerical Study of VortexShedding Suppression in Laminar Flow about a Cylinder near a Plane Boundary", Ph. D. Thesis, Dept. of Mechanical Engineering \& Materials Science, Rice University, Houston, (1989).

(2) Sarpkaya, T., "Vortex-Induced Oscillations-A Selective Review", J. Appl. Mech. Vol. 46, p. 241258 (1979).

(3) Gerrard, J. H., "The Mechanics of Formation Region of Vortices Behind Bluff Bodies", J. Fluid
Mech. Vol. 25, p. 401-413 (1966).

(4) Blevins, R. D., Flow Induced Vibrathon, Van Nostrand Reinhold Company, New York, (1977).

( 5 ) von Karman, Th., in Collected Works I, Butterworths Scientific Publications, London, p. 324-338, (1956).

(6) Allen, D. W., "Numerical Simulation of VortexInduced Oscillation of an Elastically-Mounted Circular Cylinder Using Body-Fitted Coordinates", Ph. D. Thesis, Dept. of Mechanical Engineering \& Materials Science, Rice University, Houston, (1987).

(7) Schlichting, H., Boundary Layer Theory, McGraw-Hill, New York, (1979).

( 8 ) Cohen, R. D., and Walker, W. F., "Modelling the Boundary Layer Behavior During Vortex Shedding", Int.J. Eng'g Fluid Mech. Vol. 2, p. 331-342 (1989).

( 9 ) Kim, B. K., Vanden Brink, D. J., Cramer, M. S., and Telionis, D. P., "Unsteady Convection Over Circular Cylinders", A.I.Ch.E. J. Vol. 33, p. 19-25 (1987).

(10) Blevins, R. D., Handbook of Fluid Dynamics, Van Nostrand Reinhold Company, New York, (1984).

(11) Bearman, P. W., "The Effect of Base Bleed on the Flow Behind a Two-Dimensional Model with a Blunt Trailing Edge", Aero. Quart. Vol. 18, p. 207-224 (1967).

(12) Wood, C. J., "Visualisation of an Incompressible Wake With Base Bleed", J. Fluid Mech.Vol. 29, p. 259-272 (1967).

(13) Castro, I., "Wake Characteristics of Two-Dimensional Perforated Plates Normal to an AirStream", J. Fluid Mech. 46, 599-609 (1971).

(14) Low, H. T., and Newman, B. G., "Two-Dimensional Flow Past Bluff Flexible Membranes of Low Porosity", Aeronautical J. Vol. 90, p. 313-334 (1986). 